\title{
The mDOM - A multi-PMT Digital Optical Module for the IceCube-Gen2 neutrino telescope
}

\author{
The IceCube-Gen2 Collaboration ${ }^{\dagger}$, M. Kossatz ${ }^{\ddagger}$ A. Kretzschmann ${ }^{\ddagger}$, S. Lindner ${ }^{\S}$, \\ and D. Shuklin ${ }^{\S}$ \\ ${ }^{\dagger}$ http://icecube.wisc.edu/collaboration/authors/icrc17_gen2 \\ * DESY, Platanenallee 6, D-15738 Zeuthen, Germany \\ § FAU, Cauerstraße 9, D-91058 Erlangen, Germany \\ E-mail: lew.classen@icecube.wisc.edu
}

Following the first observation of an astrophysical high-energy neutrino flux with the IceCube observatory in 2013, planning for a next-generation neutrino telescope at the South Pole, IceCubeGen2, is under way, which will significantly expand the sensitivity both towards high and low neutrino energies. The detector is envisioned to instrument $5-10 \mathrm{~km}^{3}$ of the deep clear ice with up to 10000 optical modules to detect the Cherenkov light from charged secondaries created in the interaction of neutrinos in the ice. Apart from the larger volume, a significant increase in the sensitivity is expected to originate from advanced optical modules with several concepts currently being under development. One such concept is the multi-PMT Digital Optical Module (mDOM) which, in contrast to the "conventional" layout with a single ten-inch photomultiplier tube (PMT), features 24 three-inch PMTs inside a pressure vessel pointing isotropically in all directions. This layout provides an almost uniform angular acceptance and an increased effective area by more than a factor two. Additionally, directional information on the detected photons is obtained and background can be suppressed using local coincidences while at the same time the dynamic range of the module is increased.

The contribution provides an introduction to the design as well as an overview of the current status of mDOM development and prototype construction. It also highlights further optimization potential and remaining challenges en route to a fully functional multi-PMT optical module for IceCube-Gen2.

Corresponding author: L. Classen ${ }^{* 1}$

${ }^{1}$ WWU Münster, Wilhelm-Klemm-Straße 9, D-48149 Münster, Germany

35th International Cosmic Ray Conference - ICRC2017

10-20 July, 2017

Bexco, Busan, Korea

${ }^{*}$ Speaker. 


\section{Multi-PMT optical module concept}

In contrast to the optical modules of all currently operative large volume neutrino telescopes, featuring a single large-area PMT inside a spherical pressure vessel, a multi-PMT optical module contains an array of smaller-sized PMTs. The effective segmentation of the sensitive area into individually read-out channels, first utilized in the Nevod detector [1] and introduced to the field of neutrino astronomy by the KM3NeT project [2], comes with several attractive advantages compared to the conventional single-PMT concept:

- Increase of sensitive area: The area of e.g. ten three-inch PMTs roughly equals one teninch tube. A multi-PMT module containing several ten such PMTs thus features a sensitivity equivalent to several conventional optical modules (for instance those of IceCube). As photons are the only source of information in a neutrino telescope, the collection of more photons means more information and consequently better reconstruction of event signatures assuming similar time resolution.

- Superior photon counting: The total number of arrived photons can be more directly derived from the number of hit PMTs compared to the extraction from a multi-photoelectron signal waveform. This feature can be e.g. be useful for a simple definition of cuts on the photon number by requiring a certain number of hit PMTs per module.

- Extended dynamic range: Cathode segmentation also benefits the overall dynamic range of the module, as multiple photons arriving at the same time are more likely to hit different PMTs. Assuming comparable dynamic ranges of small and large PMTs, the collective range is extended by a factor given by the number of small PMTs facing one direction.

- Improved angular acceptance: With the distribution of the sensitive area across the total solid angle, a near-homogeneous $4 \pi$ coverage is achieved. This is particularly important as the power of modern analyses is based on $4 \pi$ sensitivity [3].

- Intrinsic directional sensitivity: The orientation of the hit PMT, in combination with its known angular acceptance (see Fig. 2, b), carries additional information on the direction of the incoming photon available for event reconstruction.

- Local coincidences: Coincidences between individual PMTs of a module allow the suppression of uncorrelated background as well as the identification of low-energy neutrinos as for example generated in supernova explosions.

The hardware functionality and physics capability of the multi-PMT concept have in the meantime been demonstrated in-situ with the deployment and operation of KM3NeT prototypes in the Mediterranean [4]. Due to the development of suitable three-inch PMTs by several manufacturers these devices are meanwhile available at moderate prices resulting in the "price per photocathode area" for a multi-PMT module being comparable to, or even lower than, the value for the conventional layout.

\section{2. mDOM - a novel optical module for IceCube-Gen2}

With a substantially expanded sensitivity range, spanning from $\mathrm{GeV}$ to $\sim \mathrm{EeV}$ neutrinos, IceCube-Gen2 [5], the next-generation Antarctic neutrino telescope, is designed to identify the 
sources of the high-energy cosmic neutrinos discovered in IceCube, including a detailed spectral analysis and flavor measurement. At the lower end of its sensitivity spectrum it will address neutrino physics investigating atmospheric neutrinos. The mDOM (multi-PMT Digital Optical Module) project aims at bringing the advantages of the multi-PMT concept to the South Pole.

The design, originally based on the optical module of KM3NeT, has been under development or several years and is currently entering prototype construction phase. The completion of a first, fully functional module is scheduled for late 2017. In this prototype configuration the $\mathrm{mDOM}$ features an isotropically oriented array of 24 reflector-equipped three-inch PMTs harbored by a quasi-spherical 14 -inch borosilicate glass pressure vessel. An explosion view of the prototype layout is presented in Fig. 1 highlighting the main components. Most mechanical components have been verified individually in the lab, or currently are in the process of qualification, without the emergence of show-stoppers.

The mDOM pressure vessel will slightly deviate from the optimal spherical form factor. The design is driven by the limited diameter of the borehole and the non-reducible length of the PMTs. Based on finite-element simulations by the manufacturer, the pressure vessel is rated for 700 bar. This criterion, which is substantially larger than the hydrostatic pressure at the location of the detector, is based on the measured pressure peaks during re-freezing: up to 550 bar were observed during IceCube construction. The design was optimized for mass production with the final price

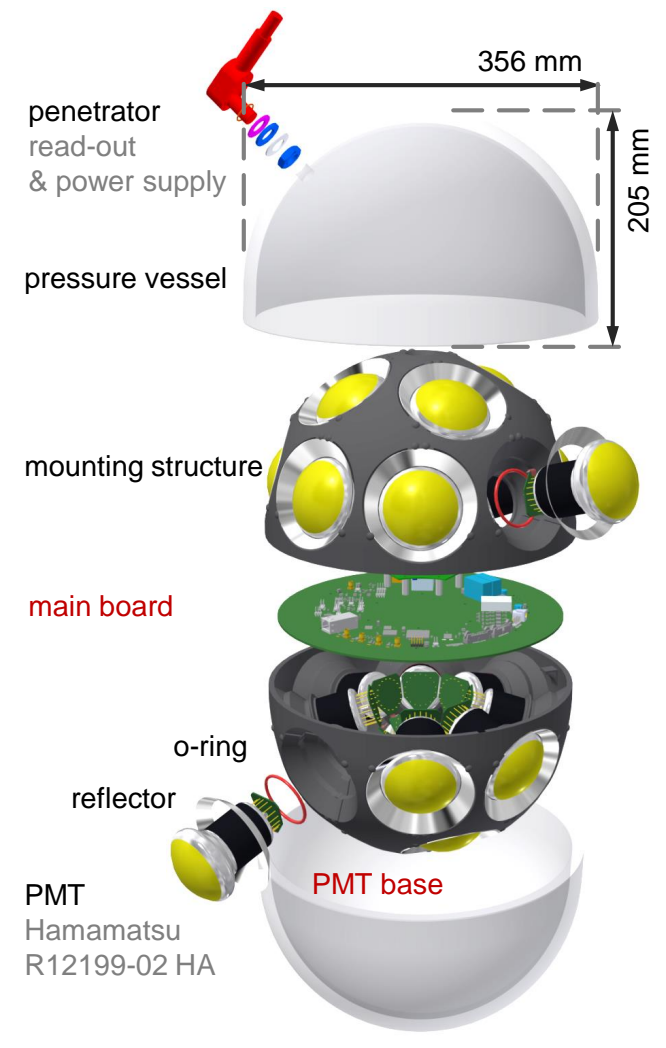

Figure 1: Default configuration of the $\mathrm{mDOM}$, featuring main components. The development of the devices marked red needs to be finalized before prototype production. not significantly deviating from comparable commercially available spherical vessels. Several specimen have been acquired from Nautilus GmbH and are currently used for tests.

Curing two-component silicone gel, poured between the PMTs and the pressure vessel, provides optical coupling as well as structural stability. The baseline choice for prototyping is QGel 900 by Quantum Silicones which has been employed in the original IceCube optical module and proven to be usable at low ambient temperatures.

Mounted around the entrance window of the PMTs, light concentrators or reflectors increase the PMT's effective area for vertical illumination. The devices are produced from enhancedreflectivity coated aluminum sheets. Considering the entire solid angle, they merely redistribute the PMT's angular acceptance, resulting in no net gain in sensitivity. In practice however, reflec- 
tors allow to reclaim photons otherwise lost to shadowing and absorption in glass and gel ${ }^{1}$. With respect to realistic configurations the utilization of reflectors yields an extra $\sim 20 \%$ in overall module sensitivity. The material ${ }^{2}$ was chosen to maximize the average Cherenkov-weighted reflectivity based on lab measurements. In a Monte Carlo study, simulating the incidence of a plane wavefront, the opening angle was optimized to provide maximum sensitivity for vertical photons, narrowing the PMT's field of view. This resulted in a final angle of $51^{\circ}$ with respect to the symmetry axis of the PMT. The effective concentration of the photon acceptance at small incidence angles comes at only marginal cost in terms overall PMT sensitivity ( $<1 \%$ deviation from the achievable maximum).

The default PMT for prototype construction is the R12199-02 HA model by Hamamatsu Photonics, which is based on the main PMT of the KM3NeT project. This model performed best during the mass screenings of quantum efficiency, transit-time spread, dark rate and the probability of spurious pulses conducted by the collaboration [6].

The PMTs low temperature operability was tested down to $-60^{\circ} \mathrm{C}$. While all other characteristics were not affected significantly, the gain and the dark rate were found to improve with cooling, with the gain increasing by some $10 \%$ and the noise rate dropping to $\sim 50 \mathrm{~s}^{-1}$ measured at a $\sim 0.3$ photoelectron threshold level. Simplifying read-out circuitry, the PMTs are operated with negative supply voltage (i.e. cathode at negative potential, anode grounded). In order to reduce the noise entailed by this choice, the PMTs will be used in a configuration where its conductively coated outer surface is placed at cathode potential (HA coating). For further noise reduction the (electrically conductive) reflectors will be connected to the photocathode potential, as floating (or even grounded) reflectors were found to promote high and unstable dark rates.

A mounting structure is used to position PMTs, reflectors and the main electronics board inside the pressure vessel. This component is produced via selective sintering (aka three-dimensional printing) from (white) polyamide and subsequently painted dull black with a non-conductive acrylic paint. The color was chosen as a white structure only marginally increases the overall module sensitivity while enhancing the amount of undesired photon scattering. In addition to positioning, the design ensures electric insulation of the photocathode with gel pockets, required by the negatively fed PMTs.

Power is supplied via twisted pair copper cables limiting the total power budget of all modules connected to one wire pair to $9.4 \mathrm{~W}$. For the mDOM a consumption of $<3 \mathrm{~W}$ is targeted, allowing to mount three modules per pair. The PMT base is therefore equipped with low-power Cockkroft-Walton circuitry for in-situ high-voltage generation based on a Nikhef design [7] as well as front-end electronics. As a detailed digitization of the PMT pulse is not feasible within the tight power constraints, in the baseline digitization concept, the analog waveform is split after pre-amplification and passed to four base-borne discriminators with independently adjustable thresholds. The four (analog) time-over-threshold (ToT) step signals from the PMT are routed via single-ended circuit board tracks to an FPGA on the main board [8], clocked at $150 \mathrm{MHz}$, where the leading and trailing edge times for each channel are determined. Parallel processing allows an effective sampling of each channel at a rate of $600 \mathrm{MHz}$. As two channels are employed in digitizing data from the lowest threshold, the resulting effective sampling is $1.2 \mathrm{GHz}$ at this level.

\footnotetext{
${ }^{1}$ The traversed layer thickness increases significantly for flatly incoming photons.

${ }^{2}$ Almeco Vega 95-100
} 
The information finally available on each pulse amounts to four time-stamped ToT values. As of now, major aspects of the electronics functionality have been successfully tested in prototype setups. In-situ high-voltage generation was found to work. The base features anode output linearity (on a $10 \%$ level) up to $\sim 500$ photoelectrons deposited in $\lesssim 1 \mathrm{~ns}$. A problem was found in spiked noise, generated on the base below $\sim 0^{\circ} \mathrm{C}$, whi ch is currently being investigated. The functionality of the multi-ToT digitization scheme was also assessed and found to work as expected. The demonstration setup included analog ToT generation, signal transport to an FPGA, as well as the digitization of the leading and trailing edge times. The signal-transit time is shown as an example of the performance in Fig. 2, d.

This output signal will be transferred to a stand-alone test bench computer for storing and analysis. Upon completion, the prototype will undergo an extensive scrutinizing program in the lab to verify its functionality and readout. In addition, in-situ qualification and calibration studies will be performed placing the mDOM inside the Nevod detector in Moscow. Simultaneous read-out of the Nevod modules will e.g. enable mDOM calibration using atmospheric muons as a source of Cherenkov light.

The expected mDOM performance was studied in Geant 4 simulations ${ }^{3}$, in particular in comparison to the properties of an alternatively proposed enhanced high quantum efficiency IceCube DOM (PDOM), providing a reference for the planned qualification tests. If not stated otherwise the properties were derived for the region of maximum ice transparency, i.e. between $200 \mathrm{~nm}$ and $600 \mathrm{~nm}$. Maximum sensitivity inhomogeneity ${ }^{4}$ across the total solid angle was found to be $\sim 10 \%$ (see also Fig. 2, a). Depending on whether a Cherenkov style $1 / \lambda^{2}$ photon spectrum or a flat one is assumed ${ }^{5}$, the mean $\mathrm{mDOM}$ sensitivity, in the prototype configuration discussed here, surpasses that of the considered single-PMT module by a factor of $\sim 2.25$ to $\sim 2.15$. This figure of merit was derived averaging the simulated effective areas, with respect to an incident plane wave, for both optical modules over the solid angle and the ice transparency window, including known respective quantum efficiencies (Fig. 2, c).

To illustrate the potential of intrinsic directionality, the capability of a single mDOM to reconstruct the direction to an isotropic photon point source was assessed. The direction to the source was determined fitting the maximum of the cumulative fields of view of all $24 \mathrm{mDOM}$ PMTs, weighted by the respective number of detected photons. In an idealized scenario ${ }^{6}$ this simple approach allows to reconstruct the direction of the source at a distance up to $100 \mathrm{~m}$ with a precision of $\lesssim 1^{\circ}$, based on a total of $4 \cdot 10^{12}$ emitted photons. A more sophisticated version of the algorithm, will allow to determine in-situ the orientation of the $\mathrm{mDOM}$ in the ice using the POCAM [9] calibration module ${ }^{7}$.

\footnotetext{
${ }^{3}$ The detailed simulation model features realistic geometries as well as optical material properties of the respective modules and was validated against lab measurements.

${ }^{4}$ Given in terms of the relative deviation form maximum acceptance.

${ }^{5}$ As UV photons experience stronger scattering in the ice the original Cherenkov spectrum becomes flatter with distance.

${ }^{6}$ Assuming homogeneous ice without dust layers featuring the properties of the relatively clean environment at a depth of $\sim 2278 \mathrm{~m}$

${ }^{7}$ The Precision Optical CAlibration Module will produce isotropic light pulses of up to $10^{10}$ photons each. Thus, the number of photons needed to achieve the discussed precision corresponds to 400 pulses only.
} 

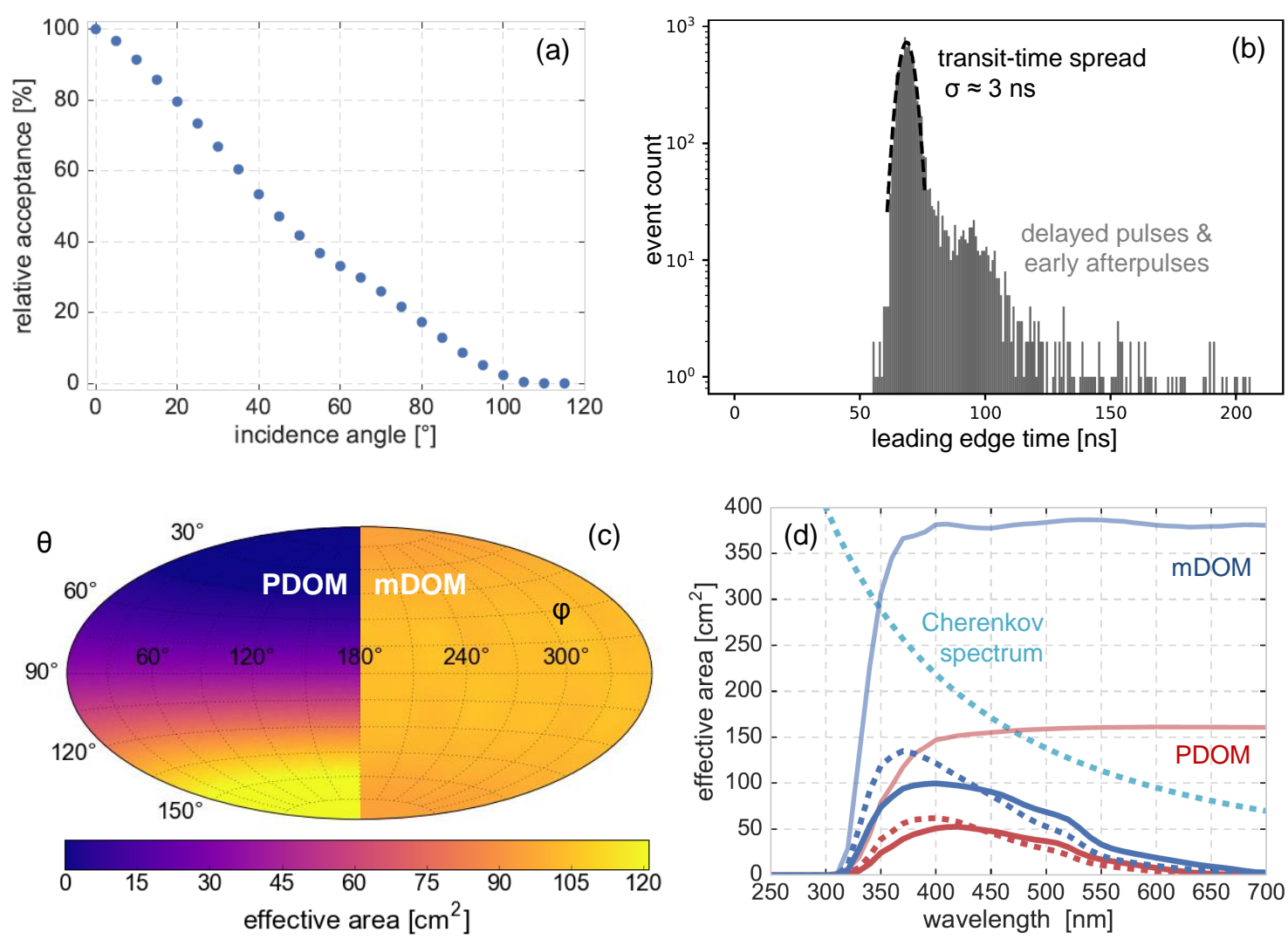

Figure 2: Module sensitivity simulated for the prototype mDOM configuration: (a) Effective area comparison between the mDOM and an high quantum efficiency single-PMT module (PDOM). (b) Angular acceptance profile of a Hamamatsu R12199-02 PMT inside the mDOM. (c) Solid-angle averaged effective areas of $\mathrm{mDOM}$ and PDOM. Spectra are shown including (solid line) and excluding (light solid line) the quantum efficiencies of the respective PMTs. Dashed lines mark the Cherenkov spectrum and respective Cherenkov-weighted effective areas in arbitrary units.

(d) Readout functionality demonstration: Single photoelectron signal transit-time distribution determined with the prototype readout consisting of PMT, four-comparator base and digitizing FPGA.

\section{Challenges and perspectives}

Although the design of the module is quite mature, some components require work before deployment in the deep ice while others have the potential to be further optimized. Together with corresponding improvement strategies they will be discussed in this section, as will additional devices not included in the prototype. Further enhancement perspectives are expected to be revealed in the course of the qualification phase.

The Vitrovex glass of the default pressure vessel surpasses the Benthos glass of the original IceCube module in terms of UV transparency and is in turn outperformed by the custom glass mixture employed in the D-Egg [10]. The gain from using these glasses was simulated to be $\sim 15 \%$ and $\sim 20 \%$, respectively. During noise investigation Vitrovex glass was found to contribute 
significantly to the background rate. In fact, due to the purity of the polar ice, glass-induced noise will be the main signal acquired by the PMTs, defining the data rate, and therefore the amount of required compression given the limited bandwidth of the copper cables foreseen in IceCube-Gen2. Background from the Benthos glass was found to be considerable lower ${ }^{8}$. In the current state of the investigations, the noise was traced back to scintillation induced by charged secondaries of the decay of trace elements with an additional smaller contribution from Cherenkov radiation. Being vital for the understanding and prediction of the final rate, the photon yield, spectrum, as well as the decay time of this scintillation are now subject to detailed investigation. While the Vitrovex glass will be used for prototype construction, alternative low-background glasses, favorable not at the cost of UV sensitivity loss, are under consideration for the final module. In addition negotiations with Nautilus $\mathrm{GmbH}$ concerning possibilities for reducing the radioactive contamination are under way. The structural stability of the component, as well as pressure-induced shrinking, will be assessed in a hyperbaric chamber.

As the current ${ }^{9}$ default optical gel, QGel 900 by Quantum Silicones, slightly reduces the module sensitivity at short wavelengths due to its transmission properties, alternative brands are under consideration. One possible choice is the custom gel developed for the D-Egg, featuring superior transmission properties, including better UV transparency, once it becomes commercially available. At the moment the default gel is investigated for chemical compatibility ${ }^{10}$ with other mDOM components, as well as for its scintillation properties, which are crucial for the estimation of its background noise contribution.

Based on the efforts of several manufactures for the KM3NeT project, fast, enhanced-area three-inch PMTs are now available from several companies at moderate cost. While the Hamamatsu PMT is currently considered the baseline, alternative models by ET Enterprises, HZC and MELZ, feature comparable performance while surpassing its photocathode area by up to $\sim 25 \%$. Their employment in the mDOM allows for an easily accessible sensitivity increase of the final module by this factor. Early 2017 saw the availability of a so-called "super-bialkali", enhanced quantum efficiency version of the Hamamatsu R12199-02 PMT featuring an average sensitivity gain of $35 \%$ to $40 \%$ with respect to the default PMT. A shortened version of the tube is now also offered, allowing to losen the tight space constraints on module electronics. Specimen of both models have been acquired and their properties are subject to detailed study at the moment.

As introduced above, the mDOM prototype will feature a four-level pulse digitization. In a more ambitious approach the analog PMT output will be fed into a custom base-borne ASIC, comprising 63 comparators in its final version thus allowing for a detailed sampling of complex waveforms. The individual comparator levels, featuring level precision of $200 \mu \mathrm{V}$ and a maximum amplitude of $\sim 1.8 \mathrm{~V}$, can be defined by combining the 255 equidistant steps resulting from an 8-bit equal resistor chain. An optimization study concerning level location is under way. The ASIC also contains a $2^{N}-1$ to $N$ encoder which reduces the data to a 6-bit output word. As in the baseline design the output is time stamped in a central FPGA. The higher level of integration will also reduce power consumption. The layout of a first reduced-performance version of the ASIC (featuring 31

\footnotetext{
${ }^{8} \mathrm{~A}$ factor of $\sim 2.5$ was found between the rates generated by Vitrovex and Benthos glass, respectively

${ }^{9}$ The originally envisaged Wacker 612 gel, used in KM3NeT, was found undergo undesired crystallization, turning hard and opaque, at low temperatures.

${ }^{10} \mathrm{~A}$ variety of materials can inhibit gel curing so that great care has to be taken here.
} 
thresholds) has been recently completed.

The space available between the individual PMTs of the mDOM is foreseen to harbor calibration devices, such as fast LED pulsers and potentially also cameras [11], which will enhance the mDOMs potential for self- as well as inter-DOM calibration.

In the final in-ice configuration the optical modules will be attached to the vertical electromechanical cable by means of metal cables and a so-called harness produced from stainless steel. It is foreseen to modify the proven IceCube "waist-band" [8] with the goal of minimum PMT shadowing. A reduction of cable mounting points from three to two is also under consideration.

Simulations of the entire future detector comparing the performance of all considered optical module alternatives, namely PDOM [8], D-Egg [10], WOM [12], and mDOM, in terms of horizontal muon reconstruction precision are under way in the collaboration [13]. As of now, the mDOM performance is consistent with its increased sensitive area throughout the considered muon energy range, while at the low end, corresponding to few $\mathrm{GeV}$, it is further enhanced by the segmentation of the cathode area.

\section{Summary}

The adaptation of the multi-PMT concept for the South Pole has resulted in the development of the mDOM for IceCube-Gen2. The project is currently entering prototype production phase expecting a fully functional prototype by the end of the year.

\section{References}

[1] V. M. Aynutdinov et al., Ap\&SS 258 (1), (1997) 105 - 116

[2] KM3NeT Coll., S. Adrián-Martínez et al., J. Phys. G43 (8), (2016) 084001 [arXiv:1601.07459]

[3] IceCube Coll., M. G. Aartsen et al., Phys. Rev. Lett. 113, (2014) 101101 [arXiv:1405.5303]

[4] KM3NeT Coll., S. Adrián-Martínez et al., Eur. Phys. J. C76 (2), (2016) 54 [arXiv:1510.01561]

[5] IceCube Coll., M. G. Aartsen et al., (2014) [arXiv:1412.5106]

[6] C. M. Mollo et al., JINST 11 (08), T08002 (2016) [arXiv: 1604.03922 ]

[7] P. Timmer, E. Heine and H. Peek, JINST 5 (12), (2010) C12049

[8] IceCube-PINGU Coll., P. Sandstrom et al., AIP Conf. Proc. 1630, (2014) 180-183

[9] IceCube-Gen2 Coll., PoS ( ICRC2017) 934 (these proceedings)

[10] IceCube-Gen2 Coll., PoS ( ICRC2017) 1051 (these proceedings)

[11] IceCube-Gen2 Coll., PoS ( ICRC2017) 1040 (these proceedings)

[12] IceCube-Gen2 Coll., PoS ( ICRC2017) 1052 (these proceedings)

[13] IceCube-Gen2 Coll., PoS ( ICRC2017) 991 (these proceedings) 